\title{
Émile Ollivier, Passaggi
}

\section{Emanuela Cacchioli}

\section{(2) OpenEdition}

\section{Journals}

\section{Edizione digitale}

URL: http://journals.openedition.org/studifrancesi/1664

DOI: 10.4000/studifrancesi. 1664

ISSN: 2421-5856

\section{Editore}

Rosenberg \& Sellier

\section{Edizione cartacea}

Data di pubblicazione: 1 novembre 2014

Paginazione: 649-650

ISSN: 0039-2944

\section{Notizia bibliografica digitale}

Emanuela Cacchioli, «Émile Ollivier, Passaggi », Studi Francesi [Online], 174 (LVIII | III) | 2014, online dal 01 novembre 2014, consultato il 18 septembre 2020. URL : http://journals.openedition.org/ studifrancesi/1664; DOI : https://doi.org/10.4000/studifrancesi.1664

\section{Questo documento è stato generato automaticamente il 18 settembre 2020.}

\section{(c) (i) $(9$}

Studi Francesi è distribuita con Licenza Creative Commons Attribuzione - Non commerciale - Non opere derivate 4.0 Internazionale. 


\title{
Émile Ollivier, Passaggi
}

\author{
Emanuela Cacchioli
}

\section{NOTIZIA}

ÉMILE OllivieR, Passaggi, trad. it. di Elena PESSINI, Piacenza, Nuova Editrice Berti, 2013, pp. 190.

1 La collana dedicata alla narrativa straniera della nuova Nuova Editrice Berti si impreziosisce di un volume di grande valore: si tratta della traduzione di Passaggi di Émile Ollivier, uno dei maggiori scrittori francofoni degli ultimi decenni. Finalmente tradotto in italiano e disponibile per tutti coloro che non conoscono il francese, Passaggi è il romanzo che riflette sull'erranza e sulla condizione transitoria dell'esistenza umana. Tema privilegiato è l'esilio, o meglio gli esili, plurimi, concreti e psicologici, con i quali ogni uomo è costretto a confrontarsi. Lo scrittore dà voce a tutti coloro che, volontariamente o per imposizione, sono costretti a lasciare la terra natale. Come è accaduto allo stesso Ollivier, haitiano di nascita, ma quebecchese di adozione dal 1964, anno in cui ha abbandonato Port-au-Prince in seguito a divergenze con il regime dittatoriale.

2 Passaggi racconta spostamenti spaziali, partenze volontarie, ritorni impossibili, residenze transitorie o definitive dettate da ragioni molto diverse. Il romanzo celebra anche i movimenti temporali verso il passato, verso l'infanzia, periodo privilegiato dell'esistenza, che rappresenta le proprie radici culturali. In quest'ultimo caso, il ritorno è impossibile perché il trascorrere degli anni ci proietta in una dimensione distante nel tempo e nello spazio che diviene irriconoscibile.

Il romanzo racconta due vicende che scorrono in parallelo nei primi capitoli per poi intrecciarsi e fondersi perché, per quanto si tratti di due situazioni completamente diverse, la condizione umana accomuna tutte le esistenze: le storie che ruotano attorno all'erranza portano lo stesso carico di speranze, di illusioni, ma anche di dolore e di sofferenza. Da un lato, troviamo Normand, intellettuale divorato dalla nostalgia che intraprende un viaggio negli ultimi giorni della sua vita per guardare dentro se stesso e 
interrogare la sua memoria. Dall'altro lato, un gruppo di haitiani che abbandona il paese natale e sfida il mare in cerca di una vita migliore. È quest'ultimo il racconto più originale: il lettore assiste alla costruzione del veliero, alla traversata, all'imperversare della tempesta e al tragico naufragio. Una storia che è profondamente diversa dalla vicenda che mette al centro Normand perché il gruppo di esuli, guidato da Amédée, rappresenta l'identità, la memoria dei riti ancestrali e della celebrazione vudù che trova una magnifica rappresentazione nella scena della possessione durante una cerimonia propiziatoria a bordo del veliero.

4 Passaggi è un romanzo che merita di essere conosciuto anche per il legame con la tradizione creola e per quel proliferare di colori, odori, sapori e di emozioni forti che emergono nitidamente dalle pagine del testo. Il lettore rimane colpito da queste sensazioni grazie ad un'ottima resa in italiano che deriva dalle preziose conoscenze che la traduttrice possiede in merito alla cultura creola. Elena Pessini ha inoltre curato la Postfazione (pp. 179-190) che conclude il romanzo e che ha il merito di introdurre il lettore profano all'interno dell'universo magnetico e coinvolgente descritto da ollivier.

5 L'augurio è che la missione della Casa Editrice Berti prosegua e che altre opere altrettanto interessanti possano trovare spazio e riconoscimento presso il pubblico italiano. 